\title{
Laser action by tuning the oscillator strength
}

\author{
Jérôme Faist, Federico Capasso, Carlo Sirtori, Deborah L. Sivco, Albert L. Hutchinson \& Alfred Y. Cho
}

Bell Laboratories, Lucent Technologies, 700 Mountain Avenue, Murray Hill, New Jersey 07974, USA

The threshold condition for laser action is usually achieved when the population difference between the initial and final energy levels of the laser transition reaches a critical value, determined by the equality between optical gain and losses. But the threshold condition can also be achieved by increasing the oscillator strength of the laser transition itself, while the population difference is held constant. This forms the basis of a new class of semiconductor lasers, a notable feature of which is broad wavelength tunability (on application of an electric field) in the technologically important mid-infrared region of the spectrum.

A particularly rich area of recent research in semiconductor nanostructures ${ }^{1}$ has been that of transitions between sub-bands in quantum wells $s^{2,3}$. These optical transitions connect electronic states within the same band (conduction or valence band) in semiconductor quantum wells, and were first investigated (in the 1960s) in the two-dimensional electron gas at the silicon/silicon dioxide interface ${ }^{4}$. These investigations were subsequently extended to heterojunction quantum wells, thus providing a major impetus to the engineering of new photonic materials and related optoelectronic devices in the mid-infrared region of the spectrum (wavelength $4-12 \mu \mathrm{m}$ ) and beyond ${ }^{2,3,5}$. Transitions between sub-bands are useful for infrared emitters and detectors ${ }^{2,3,6-10}$ because they make it possible to cover a broad range of wavelengths using the same combination of InP-based and GaAs-based state-of-the-art semiconductor alloys that are used for high-speed electronics and lightwave communications. In these devices one can design and modify at will not only the energy levels and the wavefunctions, the 'intersubband' optical matrix elements and the corresponding transition probability, but also the intersubband electron-phonon scattering rates and the electron lifetimes ${ }^{3,5,6}$. This has led to quantum-cascade lasers in which the intersubband population inversion condition is designed ${ }^{6-11}$. Inversionless lasers ${ }^{12}$ based on intersubband transitions have also been proposed ${ }^{13}$.

Here we report a systematic investigation of a new class of quantum-cascade lasers where quantum design is exploited to

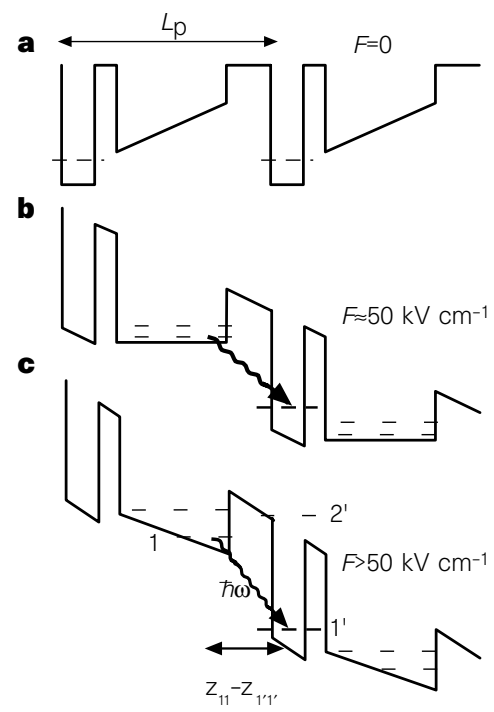

demonstrate a radically new electrical pumping scheme, oscillator strength tuning. In gas and solid-state lasers the threshold condition is achieved by increasing, by either electric or optical pumping, the population difference between the initial and final states of the radiative transition until the material gain equals the losses ${ }^{14}$. In the lasers studied here, threshold is instead achieved by keeping the population difference constant and increasing the matrix element and the energy of a suitably designed photon-assisted tunnelling transition. This transition probability tuning is an excellent illustration of how new laser materials can be designed and adds great flexibility to the engineering of semiconductor lasers in the technologically important mid-infrared spectral region.

\section{Laser action by oscillator strength tuning}

In our new laser configuration, under application of an electric field of suitable polarity (Fig. 1c), laser action takes place between the lowest energy state $(m=1)$ of one period of thickness $L_{\mathrm{p}}$ and the excited state $\left(m=1^{\prime}\right)$ of the adjacent period downstream. The electron density in the thick trapezoidal well, introduced by doping of the latter, is constant in a wide range of electric fields and temperature owing to the high injection barrier. In particular the ground state has a lifetime which, by design, is much longer (several tens of picoseconds) than that of the other states ( $\leqslant 1 \mathrm{ps})$, including the $m=1$ ' state, in the bias voltage range of the laser and contains the majority of the electron population. The electron density in this

Figure 1 Schematic energy band diagram of two periods of the new laser for different applied electric fields $F: \mathbf{a}, F=0 ; \mathbf{b}, F \approx 50 \mathrm{kV} \mathrm{cm}^{-1} ; \mathbf{c}, F>50 \mathrm{kV} \mathrm{cm}^{-}$ The electron population is concentrated in the lowest state of the large triangular well created by the applied field. Laser action occurs when the latter is increased until the oscillator strength of the $1-1^{\prime}$ transition satisfies the threshold condition (gain equals losses; c). The wavy arrow indicates the radiative transition. The horizontal dashed lines represent the energy levels and $z_{11}-z_{11^{\prime}}$, is the distance between the centroids of the electron probability densities of states 1 and $1^{\prime}$. $L_{p}$ is the length of one period. The number of periods is typically 35. 
state $N_{i}$ is therefore also essentially constant in a wide range of currents. Because the ground state $m=1$ is also the upper state of the lasing transition, the population inversion between this energy level and the empty excited state $1^{\prime}$ of the adjacent period is maintained regardless of temperature. The laser transition is based on photon-assisted tunnelling ${ }^{15-18}$ ('diagonal' transition) and its energy and matrix element can be strongly tuned by applying an electric field normal to the layers. As the bias is increased, the oscillator strength of the $\left(1-1^{\prime}\right)$ transition (which is proportional to the product of the transition energy $E_{1^{\prime} 1}$ and the modulus squared of the transition matrix element) is enhanced. This occurs by two mechanisms: (1) $E_{1^{\prime} 1}$ is shifted to higher values owing to the increased potential drop between the two states (linear Stark effect); (2) the transition matrix element is increased due to the enhanced spatial overlap (tunnelling) between the states.

When the oscillator strength and the attendant gain cross-section $\sigma_{11^{\prime}}$ (proportional to the oscillator strength) is such that the gain $\sigma_{11} N_{1}$ equals the optical losses, the threshold condition for laser action is achieved. This laser action by oscillator strength tuning is the most fundamental aspect of this new class of lasers and sets it apart from all other mechanisms of achieving threshold so far demonstrated. We have built devices lasing at photon energies corresponding to optical transitions from the ground state (Fig. 1c) for different values of the electric field, thus providing direct evidence of laser action by oscillator strength tuning. In a suitably designed structure we have demonstrated a a
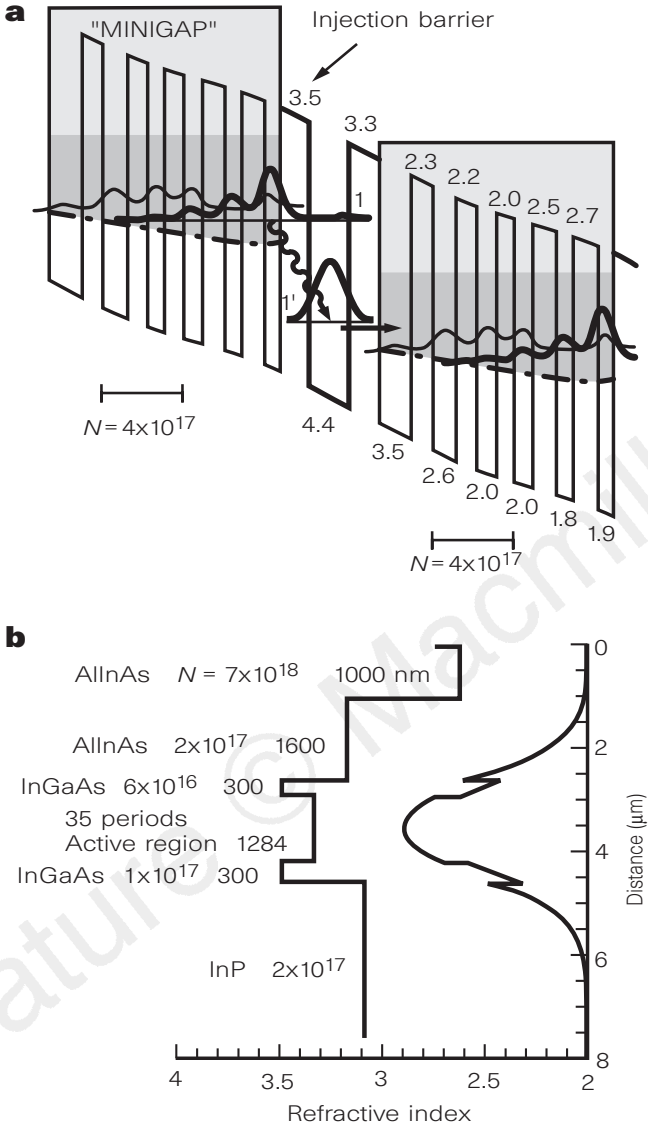

Figure 2 Schematics of the $A I_{0.48} \mid n_{0.52} A s / G_{0.47} I_{0.53} A s$ laser heterostructure D2190. The thicknesses of the layers are given in $\mathrm{nm}$ and the doping level is given in $\mathrm{cm}^{-3}$. a, Self-consistent calculation of the conduction-band diagram of a portion of the active region at an average electric field of $8.5 \times 10^{4} \mathrm{~V} \mathrm{~cm}^{-1}$. The dot-dashed line is the effective conduction-band edge of the superlattice alloy. The wavy line indicates the transition responsible for laser action. The moduli squared of the relevant envelope wavefunctions are shown. Indicated as 'minigap' are the regions with a vanishing density of states: the horizontal segment indicates the doped portion of each period where $N$ is the donor density in $\mathrm{cm}^{-3}$ The material parameters are: conduction band discontinuity $\Delta E_{c}=0.52 \mathrm{eV}$; electron effective mass $m_{\mathrm{e}}^{*}\left(\mathrm{Ga}_{0.47} \ln _{0.53} \mathrm{As}\right)=0.043 m_{0}$, where $m_{0}$ is the free electron mass and $m_{\mathrm{e}}^{*}\left(\mathrm{Al}_{0.48} \mathrm{In}_{0.52} \mathrm{As}\right)=0.078 m_{0}$. Non-parabolicities were taken in account using the method of ref. 20 with the non-parabolicity coefficient $\gamma=1.13 \times 10^{-18} \mathrm{~m}^{2}$. b. From left to right: composition profile, doping profile, refractive index and mode profile of the waveguide in the direction perpendicular to the layers. The interfaces between the AllnAs and InGaAs regions are graded in composition over a distance of $\sim 30 \mathrm{~nm}$ to smooth out the conduction-band barrier.

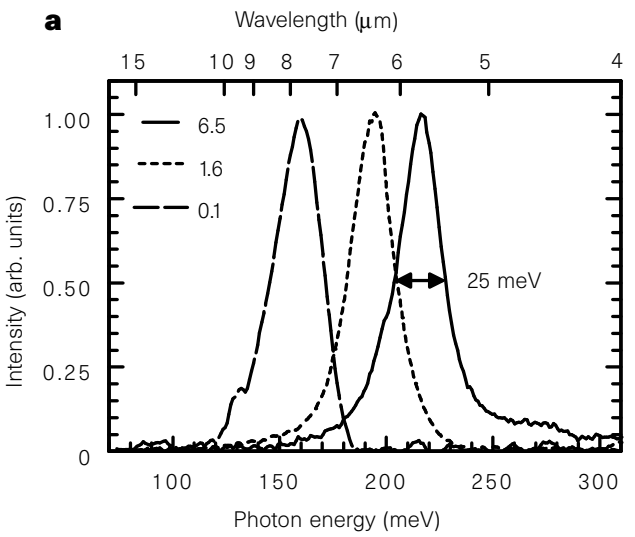

b

Current density $\left(\mathrm{KA} \mathrm{cm}^{-2}\right)$

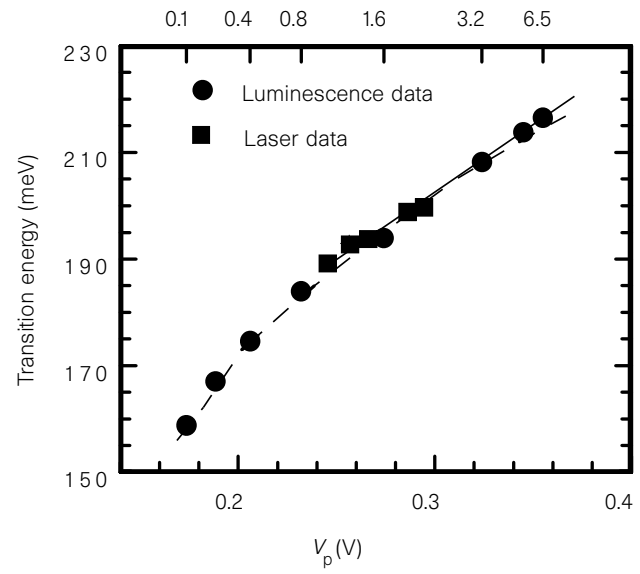

Figure $\mathbf{3}$ a, Intersubband electroluminescence spectra of sample D-2146 associated with the optical transition from the ground state $m=1$ to $1^{\prime}$ ' (see Fig. 1c) for increasing current densities, shown in key in units of $\mathrm{kA} \mathrm{cm}^{-2}$; solid line, $6.5 \mathrm{kA} \mathrm{cm}^{-2}$; short-dashed line, $1.6 \mathrm{kA} \mathrm{cm}^{-2}$; long-dashed line, $0.1 \mathrm{kA} \mathrm{cm}^{-2}$. Sample temperature, $10 \mathrm{~K}$. b. Position of the electroluminescence peak as a function of applied voltage per period $V_{p}$. The corresponding injected current density is displayed on the top horizontal axis. The straight solid line represents the regime in which the electron density in the $m=1$ state is constant and equal to the charge introduced by doping. The photon energy is then proportional to the voltage drop between the centroids of the probability densities of states 1 and $1^{\prime}$ (the linear Stark effect). Sample temperature, $10 \mathrm{~K}$. Filled circles, luminescence sample: filled squares, emission photon energies of five lasers from sample D2190 with different lengths and widths ranging from 3 to $1.5 \mathrm{~mm}$ and from 18 to $10 \mu \mathrm{m}$, respectively, corresponding to different $V_{\mathrm{p}}$. The coincidence of the laser photon energies with the optical transitions from the $m=1$ ground state is direct evidence of lasing by oscillator strength tuning. The dashed line is the result of a rigorous calculation including screening effects; the solid line is a linear approximation to the theory (equation (2)) valid when screening is negligible. 
large tuning of the wavelength based on the Stark shift of the optical transition.

The present laser differs in significant ways from previous quantum-cascade lasers $^{6-10}$. In the latter devices, the injection is by resonant tunnelling from the ground state of the injector into an excited state of a coupled or single ${ }^{11}$ quantum-well active region; the population difference between this energy level and a lower one is increased until threshold is reached. Resonant optical phonon scattering from the final state of the laser transition plays an important role in achieving population inversion in the coupledwell active region structures ${ }^{6-10}$. In addition, in these quantumcascade the gain spectrum is not electric-field tunable. Note that in the lasers with single-well active regions, population inversion is difficult to achieve because the upper-state lifetime is very short ${ }^{11}$.

\section{Laser design and electroluminescence}

The transport for increasing applied electric fields can be understood schematically from Fig. 1. At low applied electric fields, the graded region creates a thick effective barrier between the ground states of the wide GaInAs quantum wells (dotted lines in Fig. 1a), and the current flowing through the structure by hopping is negligible. A significant current starts to flow in the structure only for applied fields $F$ larger than $\sim 50 \mathrm{kV} \mathrm{cm}^{-1}$ (corresponding to a bias per period of $0.18 \mathrm{~V}$ ) necessary to compensate the conductionband gradient of opposite sign in the trapezoidal wells (Fig. 1b). In the range of fields considered $\left(F>50 \mathrm{kV} \mathrm{cm}^{-1}\right)$, the ground state $(m=1)$ of the injector is energetically far away from the ground state $1^{\prime}$ and the excited state $2^{\prime}$ of the well in the adjacent period downstream (Fig. 1c). This prevents resonant tunnelling injection into states $1^{\prime}$ and $2^{\prime}$ and the attendant negative differential resistance. Transport can then be described in terms of phonon-assisted tunnelling ${ }^{15,19}$ between states 1 and $1^{\prime}$. This ensures stable injection without high field domains in the whole operating range of our laser, unlike in the resonant tunnelling optical amplifier structures proposed by Kazarinov and Suris ${ }^{15}$. In this field range, the compositionally graded region forms a triangular well, the ground state $(m=1)$ of which contains most of the electron population $(>95 \%)$ at $10 \mathrm{~K}$ and is long-lived (tens of picoseconds) compared to other levels. As shown schematically in Fig. 1, and in more detail in Fig. 2a, one period of our structure comprises a compositionally graded well consisting of a short-period $\mathrm{Al}_{0.48} \mathrm{In}_{0.52} \mathrm{As} / \mathrm{Ga}_{0.47} \mathrm{In}_{0.53} \mathrm{As}$ superlattice (that is, a pseudo-alloy), and of $\mathrm{G} \mathrm{Ga}_{0.47} \mathrm{In}_{0.53}$ As quantum well with two $\mathrm{Al}_{0.48} \mathrm{In}_{0.52}$ As tunnelling barriers. Under applied bias (Fig. 1c), photon-assisted tunnelling emission occurs across the injection barrier upstream from the $\mathrm{Ga}_{0.47} \mathrm{In}_{0.53}$ As quantum well. The barrier downstream allows fast electron escape into the next well by tunnelling.

The layer sequence of one laser sample (D-2190) is shown in Fig. 2. Thirty-five periods of the active region, grown by molecular

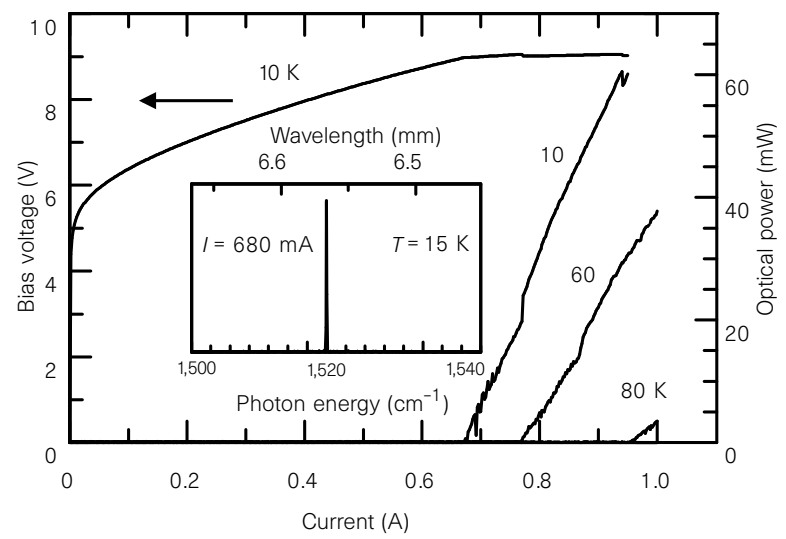

beam epitaxy (MBE) lattice-matched to an InP substrate, are inserted in an optical waveguide similar to those of quantumcascade lasers ${ }^{6-11}$. Three other laser samples (D-2152, D-2187, D-2197), based on the scheme shown in Fig. 1 but designed with slightly different parameters, were grown and displayed similar laser performance.

To study the nature of the photon-assisted tunnelling transition $\left(1-1^{\prime}\right)$, we investigated intersubband electroluminescence as a function of applied electric field. To suppress optical feedback and gain, we investigated a sample (D-2146) which has the same active region band diagram of the laser sample D-2190, but with a fewer number of periods (five) and without waveguide confining layers. To minimize free-carrier absorption in the substrate, the five periods of the active region-sandwiched between doped $\left(n^{+}=2 \times 10^{18} \mathrm{~cm}^{-3}\right)$ GaInAs contact layers-were grown on semi-insulating InP substrates. The samples were then processed into $125-\mu \mathrm{m}$-diameter circular mesas and the light coupled out through a polished wedge at a $45^{\circ}$ angle from the plane of the layers. The electroluminescence spectrum was measured at low temperature with a Fourier-transform infrared spectrometer (FTIR; ref. 17). A few spectra at selected current densities are shown in Fig. 3a.

The large spatial difference $z_{11}-z_{1^{\prime} 1^{\prime}}$ between the coordinates of the centroids of the electron probability distributions of states 1 and $1^{\prime}$, and the absence of anticrossing between the $m=1$ state and any resonance of the adjacent well, cause a strong first-order Stark shift of the $1-1^{\prime}$ transition. Accordingly, the measured electroluminescence peak shifts from $7.8 \mu \mathrm{m}$ at low current densities $\left(0.1 \mathrm{kA} \mathrm{cm}^{-2}\right)$ to $5.8 \mu \mathrm{m}$ at $6.5 \mathrm{kA} \mathrm{cm}^{-2}$. Diagonal transitions are more sensitive to interface layer roughness than vertical ones; therefore the measured full-width at half-maximum of the luminescence peak $(2 \gamma=25 \mathrm{meV}$, independent of applied bias $)$ is significantly larger than that observed $(2 \gamma=14 \mathrm{meV})$ for a vertical transition structure at similar wavelengths $s^{7,10,11}$.

The measured transition energy $E_{1^{\prime} 1}$ of the electroluminescence is plotted as a function of the voltage drop per period $V_{\mathrm{p}}$ in Fig. $3 \mathrm{~b}$ and compared to the calculated value (dashed line) using a rigorous selfconsistent calculation based on Schrödinger's and Poisson's equations ${ }^{20}$. An example of a calculated energy diagram is shown in Fig. 2a. The agreement is excellent over the whole bias range. The Stark shift of the $1-1^{\prime}$ transition $\left(\Delta E_{1^{\prime} 1}\right)$ can be written as

$$
\Delta E_{1^{\prime} 1}=q \Delta F\left[z_{11}-z_{1^{\prime} 1^{\prime}}\right]
$$

where $q$ is the electron charge and $\Delta F$ is increment of the electric field in the region between states 1 and $1^{\prime}$, neglecting the weak electric-field dependence of $z_{11}-z_{1^{\prime} 1^{\prime}}$. In the high-field regime, when the electron density is essentially all concentrated in the ground state $m=1$, for $V_{\mathrm{p}}>0.22 \mathrm{~V}, \Delta F$ can be expressed as $\Delta V_{\mathrm{p}} / L_{\mathrm{p}}$ where $\Delta V_{\mathrm{p}}$ is the voltage increment across one period.

Figure 4 Applied bias and measured continuous-wave optical power from a single facet as a function of injected current for a representative laser of wafer D2190 at various temperatures. The collection efficiency of the detection apparatus is $50 \%$. The bias voltage is only reported for the lowest temperature (10 K). The device is $3 \mathrm{~mm}$ long and $14 \mu \mathrm{m}$ wide. Inset, laser spectrum slightly above threshold $(I=0.68 \mathrm{~A})$ at $T=10 \mathrm{~K}$. 
Equation (1) then reduces to, in differential form,

$$
\frac{1}{q} \frac{\mathrm{d} E_{1^{\prime} 1}}{\mathrm{~d} V_{\mathrm{p}}} \approx \frac{z_{11}-z_{1^{\prime} 1^{\prime}}}{L_{\mathrm{p}}}
$$

Taking $z_{11}-z_{1^{\prime} 1^{\prime}}=10 \mathrm{~nm}$ and $L_{\mathrm{p}}=36.7 \mathrm{~nm}$, we find a slope $(1 / q)\left(\mathrm{d} E_{1^{\prime} 1} / \mathrm{d} V_{\mathrm{p}}\right)=0.26$ (solid line in Fig. 3) which is in good agreement with the measured value $(0.278)$ obtained from the data of Fig. $3 \mathrm{~b}$ between $V_{\mathrm{p}}=0.25 \mathrm{~V}$ and $V_{\mathrm{p}}=0.32 \mathrm{~V}$. Note that at a high bias, the theoretical curve slightly deviates from the form given by equation (2). This is due to the small electric-field-induced spatial shifts of states 1 and $1^{\prime}$, that is $z_{11}-z_{1^{\prime} 1^{\prime}}$ decreases with field.

At low bias the experimental Stark shift $(1 / q)\left(\mathrm{d} E_{1^{\prime} 1} / \mathrm{d} V_{\mathrm{p}}\right)=0.54$ is larger than predicted by equation (2). Physically, in this regime, the electron charge progressively transfers from the doped regions of the trapezoidal well into the $m=1$ state. This transfer tends to screen the field in this region and correspondingly increases the electric field in the undoped region between states 1 and $1^{\prime}$ by an amount $\Delta F$ greater than $\Delta V_{\mathrm{p}} / L_{\mathrm{p}}$. This screening regime is correctly described by our self-consistent model (dashed curve in Fig. 3b).

In luminescence structures or in lasers below threshold, radiative transitions have a negligible effect on the current density $J$ which can be written as $J=q N_{1} / \tau_{1}$ where $\tau_{1}$ is the lifetime of the $m=1$ state controlled by optical phonon emission and $N_{1}$ is its electron sheet density. In the current density range of our experiments (up to $3 \mathrm{kA} \mathrm{cm}^{-2}$ ), the injected electron density $J \tau_{1^{\prime}} / q \leqslant 2 \times 10^{10} \mathrm{~cm}^{-2}$, where $J$ is the measured current density and $\tau_{1^{\prime}} \approx 1$ ps is the lifetime of state $m=1^{\prime}$, is small compared to that of the ground state $N_{1}=3.6 \times 10^{11} \mathrm{~cm}^{-2}$, thus preventing space-charge build-up. The absence of space-charge injection in our structure implies global charge neutrality and the same field profile in each period; $n_{1}$ is therefore constant and corresponds to the charge introduced by the doping of the injection region $\left(N_{1}=3.6 \times 10^{11} \mathrm{~cm}^{-2}\right)$. As the applied field is increased, $\tau_{1}$ progressively decreases as the wavefunction of state 1 is 'pushed' inside the injection barrier by the progressive narrowing of the triangular well. This variation of $\tau_{1}$ with constant population $N_{1}$ controls the current-voltage characteristic of the device. With $N_{1}=3.6 \times 10^{11} \mathrm{~cm}^{-2}$ (sample D-2146), a lifetime $\tau_{1}$ of tens of picoseconds (ref. 21) leads to current densities $J$ in the range $1-3 \mathrm{kA} \mathrm{cm}^{-2}$.

The increase of the penetration of the wavefunction into the injection barrier with increasing field, responsible for the decrease of $\tau_{1}$, leads also to a concomitant increase of the optical matrix element $z_{11^{\prime}}$.

\section{Laser characterization}

The lasers were processed into ridge waveguides of width 10-18 $\mu \mathrm{m}$ at their bases and aligned along the $[1 \overline{1} 0]$ crystallographic direction by wet chemical etching $\left(\mathrm{H}_{3} \mathrm{PO}_{4}: \mathrm{H}_{2} \mathrm{O}_{2}: \mathrm{H}_{2} \mathrm{O}, 1: 2: 4\right)$. A 350-nmthick $\mathrm{Si}_{3} \mathrm{~N}_{4}$ insulation was deposited by chemical vapour deposition. Non-alloyed Ti/Au ohmic contacts were evaporated onto the top layer and the substrate. The lasers were cleaved in bars $1-3.5 \mathrm{~mm}$ long and the facets left uncoated. They were then soldered

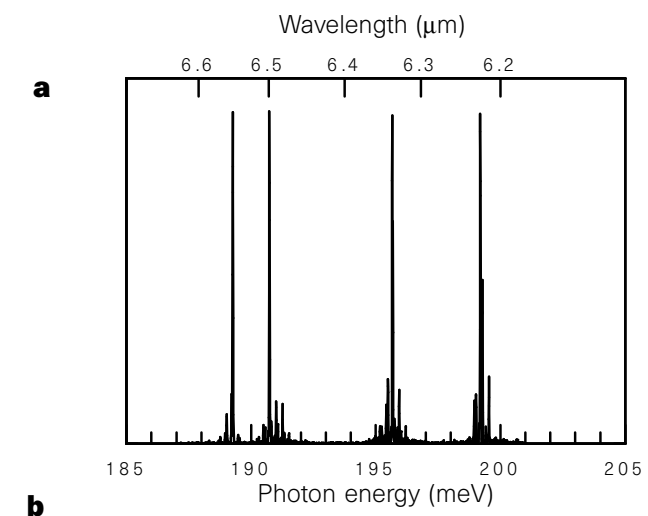

b

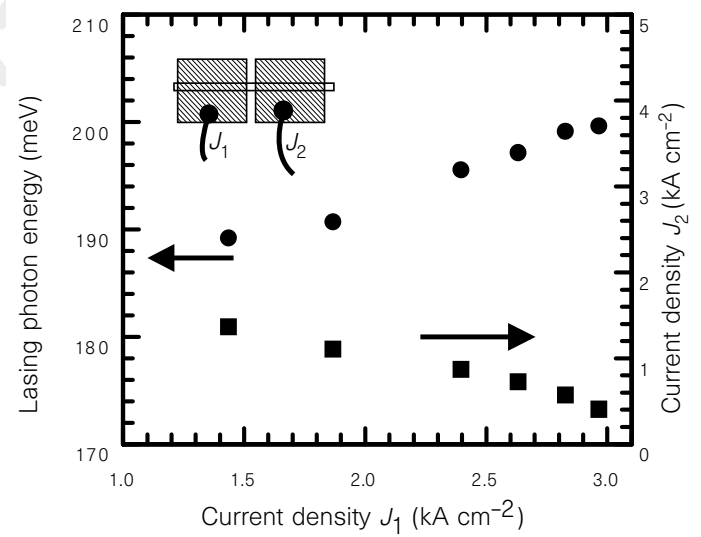

Figure 5 Tunable operation of the laser D-2190. The top metal contact is split into two sections to allow independent electrical injection of two currents densities $J_{1}$ and $J_{2}$ in the laser waveguide (see inset). The measurements are performed with the laser driven in pulsed mode above threshold with a constant peak optical power of $1 \mathrm{~mW}$. a, Measured optical spectra for different values of the currents $U_{1}$ $J_{2}$ ) from left to right, in units of $\mathrm{kA} \mathrm{cm}^{-2}$ : $(1.4,1.4) ;(1.9,1.1) ;(2.4,0.9) ;(2.8,0.6)$. b, Laser photon energy (filled circles) and current density in the second section (filled squares) as a function of injected current density in the first section.

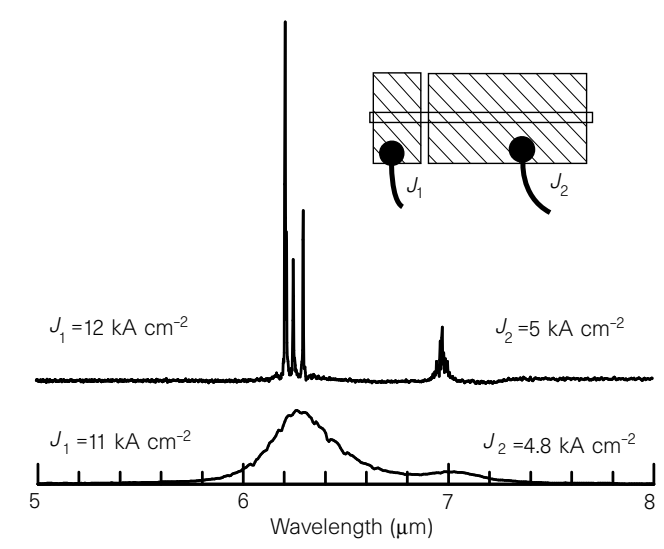

Figure 6 Two-wavelength operation of sample D-2197. The layer sequence of one period of the active region, in nanometres from left to right and starting from the injection barrier (as in Fig. 2a) is 3.5/4.4/3.5/2.8/2.2/2.2/2.0/2.0/2.5/2.1/3.2/2.2. The barrier layers are in bold; the underlined thickness correspond to the Sidoped layers $\left(N=6 \times 10^{17} \mathrm{~cm}^{-3}\right)$. The length of the small section of the waveguide is $0.8 \mathrm{~mm}$ (responsible for the short-wavelength emission) and the long section (responsible for the long-wavelength emission) is $2.8 \mathrm{~mm}$. The lower and upper curves are taken below threshold and at threshold, respectively. 
epilayer-up on copper holders, wire-bonded and mounted on a temperature-controlled $(10-300 \mathrm{~K})$ cold head of a helium flow cryostat.

Figure 4 shows the continuous-wave optical power versus drive current from a single facet of sample D-2190, obtained using f/0.8 optics and a calibrated, room-temperature HgCdTe detector. The laser operates up to $80 \mathrm{~K}$, with up to $40 \mathrm{~mW}$ of optical power at $60 \mathrm{~K}$. At $10 \mathrm{~K}$, lasing threshold is obtained at current $I=0.675 \mathrm{~A}$ and corresponds to a threshold current density $J_{\text {th }}=1.6 \mathrm{kA} \mathrm{cm}^{-2}$. This threshold density is comparable to the best results obtained in previous quantum-cascade lasers with coupled-well active region. The main reason for this is that the decrease of the matrix element of the optical transition and the increase of the transition linewidth is compensated by a much longer electron lifetime.

The voltage applied to this device is displayed in Fig. 4 as a function of injection current at $10 \mathrm{~K}$; the threshold voltage is $9 \mathrm{~V}$. Above threshold, the gain is equal to the total optical losses, pinning the bias voltage at approximately its threshold value, as shown experimentally by the plateau in the voltage-current curve above $0.675 \mathrm{~A}$. Because of this pinning, the laser wavelength does not change significantly as a function of current above threshold. However, because of the strong dependence of the transition energy on the current (Fig. 3), the laser wavelength is expected to vary considerably with threshold current density in devices of different cavity length. More specifically, because the threshold current depends on the cavity loss, the laser photon energy (which is a linear function of voltage) will vary for devices with different lengths and waveguide losses. In Fig. 3, we show (filled squares) the laser photon energy for different values of the threshold bias per period corresponding to different devices with different cavity lengths and widths. The values are in very good agreement with the luminescence measurements. For all the devices, the threshold bias per period is larger than $0.22 \mathrm{~V}$, implying that they operate in the regime of negligible screening. This measurement confirms the peculiar nature of this laser where the threshold condition is met by increasing the optical matrix element with increasing electric field while keeping constant the population of the upper state of the lasing transition. The absence of an independent 'reservoir' of electrons might have important implications for the dynamical properties of the laser and for the statistical properties of its stimulated emission.

The spectrum of a device operating in continuous wave above threshold is displayed in Fig. 4 inset. The spectrum is monomode, with a lidewidth limited by the resolution of our FTIR spectrometer $\left(0.09 \mathrm{~cm}^{-1}\right)$. Unlike quantum-cascade lasers based on a 'vertical' transition $^{22}$, these devices tend to become multimode when the power exceeds about a milliwatt. This tendency towards multimode operation is consistent with the large non-homogenous broadening of diagonal transitions.

The waveguide loss $\alpha_{\mathrm{w}}=30 \mathrm{~cm}^{-1}$ was measured by an analysis of the subthreshold luminescence ${ }^{22}$. The structure is designed to minimize intersubband absorption from the ground state $m=1$ to the excited states of the same period by avoiding resonance at the laser photon energy. We attribute the waveguide loss to residual intersubband absorption and to free-carrier absorption. At and above threshold, the peak gain equals the losses:

$$
\Gamma G_{\mathrm{p}}=\alpha_{\mathrm{w}}-\frac{1}{L} \ln (R)
$$

where $\Gamma=0.52$ is the mode overlap factor. The second term on the right-hand side of equation (3) represents the mirror losses $\alpha_{\mathrm{m}}$ where $R=0.26$ is the facet reflectivity and $L=3 \mathrm{~mm}$ is the cavity length of the relatively long device of Fig. 4 . The peak material gain $G_{\mathrm{p}}$ is a function of the matrix element $z_{11^{\prime}}(J)$ of the laser transition ${ }^{7}$

$$
G_{\mathrm{p}}=\frac{4 \pi q^{2}}{\epsilon_{0} n} \frac{z_{11^{\prime}}^{2}(J) N_{1}}{L_{\mathrm{p}}(2 \gamma) \lambda_{\mathrm{p}}}
$$

where $\lambda_{\mathrm{p}}=6.5 \mu \mathrm{m}$ is the peak emission wavelength, $n=3.2$ is the material's refractive index and $\epsilon_{0}$ is the vacuum permittivity. The matrix element $z_{11^{\prime}}(J)$ increases with current density $J$, as previously discussed, up to its threshold value $z_{11^{\prime}}\left(J_{\text {th }}\right)=0.39 \mathrm{~nm}$ obtained from equations (3) and (4). The calculated matrix element at the electric field corresponding to the pulsed threshold $J_{\text {th }}=1.4 \mathrm{kA} \mathrm{cm}^{-2}$ is $z_{11^{\prime}}=0.41 \mathrm{~nm}$, in good agreement with the previous value.

The laser D-2190 operates in pulsed mode up to temperatures of $220 \mathrm{~K}$ with a weak temperature dependence of the threshold current, a characteristic of a laser based on intersubband transitions ${ }^{9,10}$. Sample D-2197, based on a slightly different active region with a larger number of periods (45), lased close to room temperature $(280 \mathrm{~K})$ giving tens of milliwatts of pulsed optical power.

\section{Wavelength tuning and dual wavelength operation}

In the structures of our devices, the top contact metallization is not continuous along the whole waveguide but consists of segments separated by $\sim 20 \mu \mathrm{m}$, without etching or cleaving the waveguide, which are contacted in parallel. The resistance between individual sections $(\sim 200 \Omega)$ is much larger than the differential resistance of the device, allowing us to inject different current densities in different sections of the device. Let us consider a device in which the waveguide of length $L=3 \mathrm{~mm}$ is divided in two sections of respective length $a L$ and $(1-a) L$. The specific device considered here has two sections of equal length $(a=0.5)$ (Fig. 5b). In such a structure, keeping the gain constant at its threshold value is only a global requirement, that is the gain in each section $\left(G_{1}\right.$ and $\left.G_{2}\right)$ can be independently adjusted as long as the total gain is equal to the total losses

$$
\Gamma\left[\operatorname{agJ}_{1} \mathcal{L}\left(J_{1}, \lambda\right)+(1-a) g J_{2} \mathcal{L}\left(J_{2}, \lambda\right)\right]=\alpha_{\mathrm{w}}-\ln (R) / L
$$

where $\mathcal{L}\left(J_{1}, \lambda\right)$ is the lineshape factor and the quantity in parenthesis is the sum of the gains in the two sections. By injecting different current densities $J_{1}$ and $J_{2}$ in each section, the laser photon energy can now be electrically adjusted ('tuned') towards larger energies, approximately tracking the peak of the gain spectrum of the section with the larger injected current as the longitudinal model separation $\left(0.5 \mathrm{~cm}^{-1}\right)$ is negligible compared to the tuning range of the gain spectrum $\left(100 \mathrm{~cm}^{-1}\right)$. In Fig. 5, the measured photon energy and current at threshold in the second section is plotted as a function of the current in the first section. To minimize heating effects, the measurement was performed in pulsed operation. The tuning range shown in Fig. 5 for this laser (6.6$6.2 \mu \mathrm{m}$, corresponding to $\left.100 \mathrm{~cm}^{-1}\right)$ is significant for spectroscopic applications.

When the two sections have a significantly different length (that is, for $a<0.3$ in our case), a striking effect occurs: the structure is able to lase at two wavelengths simultaneously. Having widely different lengths, the two sections will individually reach threshold for a very different current density, and therefore at a very different wavelength because of the Stark tuning. It allows the gain spectrum to display two local maxima which can be brought simultaneously to threshold. We have demonstrated this effect in sample D-2197; Fig. 6 shows sub-threshold luminescence curves for increasing current in the two sections. The two maxima are clearly apparent at wavelengths 7 and $6.2 \mu \mathrm{m}$. Because of gain saturation, we were not able to drive the short section clearly above threshold and the curve at the higher current is taken at threshold.

We have demonstrated a new class of mid-infrared lasers. The importance of the mid-infrared spectrum stems for the existence of atmospheric transmission windows at 3-5 $\mu \mathrm{m}$ and $8-13 \mu \mathrm{m}$. Many gases and vapours have pronounced absorption features in these regions. Thus suitable single-frequency and tunable laser sources in this spectral range can be used for trace-gas analysis and pollution monitoring ${ }^{23,24}$. The wide tunability of these devices makes them appealing for applications such as spectroscopy of liquids which 
have broad absorption lines. Future work will concentrate on increasing the maximum operating temperature and tuning range. The electrical-to-optical power conversion efficiency of these and previous quantum cascade lasers is inherently small $(\sim 1 \%)$ owing to the high operating currents and voltages.

Received 8 January; accepted 29 April 1997.

1. Weisbuch, C. \& Vinter, B. Quantum Semiconductor Structures (Academic, San Diego, 1991).

2. Liu, H. C., Levine, B. F. \& Anderson, J. Y. Quantum Well Intersubband Transitions: Physics and Devices (NATO ASI Ser. E, Vol. 270, Plenum, New York, 1994).

3. Rosencher, E., Vinter, B. \& Levine, B. F. (eds) Intersubband Transitions in Quantum Wells (NATO ASI Ser. B, Vol. 288, Plenum, New York, 1992).

4. Ando, T., Fowler, A. B. \& Stern, F. Electronic properties of two-dimensional systems. Rev. Mod. Phys. 54, 437-672 (1982).

5. Capasso, F., Faist, J. \& Sirtori, C. Mesoscopic phenomena in semiconductor nanostructures by quantum design. J. Math. Phys. 37, 4775-4792 (1996).

6. Faist, J. et al. Quantum cascade laser. Science 264, 553-556 (1994).

7. Faist, J. et al. Vertical transition quantum cascade laser with Bragg confined excited state. Appl. Phys. Lett. 66, 538-540 (1995).

8. Sirtori, C. et al. Long wavelength $(\lambda \sim 11 \mu \mathrm{m})$ quantum cascade lasers. Appl. Phys. Lett. 69, 2810-2812 (1996).

9. Sirtori, C. et al. Mid-infrared $(8.5 \mu \mathrm{m})$ semiconductor laser operating at room temperature. IEEE Photon. Technol. Lett. 9, 294-297 (1997).

10. Faist, J. et al. High power mid-infrared $(\lambda \sim 5 \mu \mathrm{m})$ quantum cascade lasers operating above room temperature. Appl. Phys. Lett. 68, 3680-3682 (1996).

11. Faist, J. et al. Quantum cascade lasers without intersubband population inversion. Phys. Rev. Lett. 76, 411-414 (1996).
12. Harris, S. E. Lasers without inversion: interference of lifetime-broadened resonances. Phys. Rev. Lett. 62, 1033-1036 (1989)

13. Imamoglu, A. \& Ram, R. J. Semiconductor lasers without population inversion. Opt. Lett. 19, 17441746 (1994).

14. Sargent, M., Scully, M. O. \& Lamb, W. E. Laser Physics (Addison Wesley, New York, 1977).

15. Kazarinov, R. F. \& Suris, R. A. Electric and electromagnetic properties of semiconductors with a superlattice. Fiz. Tekh. Poluprov. 6, 148-159 (1972); transl. in Sov. Phys. Semicond. 6, 120-131 (1972).

16. Keay, B. J. et al. Photon-assisted electric field domains and multiphoton-assisted tunneling in semiconductor superlattices. Phys. Rev. Lett. 75, 4098-4101 (1995).

17. Faist, J. et al. Mid-infrared field-tunable intersubband electroluminescence at room temperature by photon-assisted tunneling in coupled-quantum wells. Appl. Phys. Lett. 64, 1144-1146 (1994).

18. Caine, E. J., Subbanna, S., Kroemer, H., Merz, J. L. \& Cho, A. Y. Staggered-lineup heterojunctions as sources of tunable below-gap radiation: Experimental verification. Appl. Phys. Lett. 45, 1123-1125 (1984).

19. Oberli, D. Y. et al. Direct measurement of resonant and nonresonant tunneling times in asymmetric coupled quantum wells. Phys. Rev. B 40, 3028-3031 (1989).

20. Sirtori, C., Capasso, F., Faist, J. \& Scandolo, S. Nonparabolicity and a sum rule associated with boundto-bound and bound-to-continuum intersubband transitions in quantum wells. Phys. Rev. B 50, 8663-8674 (1994).

21. Price, P. J. Electron transport in polar heterolayers. Surf. Sci. 113, 199-210 (1982).

22. Faist, J. et al. Continuous wave operation of quantum cascade lasers based on vertical transitions at $\lambda=$ $4.6 \mu \mathrm{m}$. Superlattice Microstructures 19, 337-345 (1996).

23. Martinelli, R. U. Mid-infrared wavelengths enhance trace-gas sensing. Laser Focus World 32, 77-81 (1996).

24. Tacke, M. New developments and applications of tunable IR lead salt lasers. Infrared Phys. Technol. 36, 447-463 (1995).

Acknowledgements. We thank S. N. G. Chu for transmission electron microscopy work on some of the structures, and C. Gmachl for discussions.

\section{KNOW YOUR COPY RIGHTS}

$R$ E S P E C T

O U R S

$T_{\text {he publication you are }}$ reading is protected by copyright law. This means that the publisher could take you and your employer to court and claim heavy legal damages if you make unauthorised photocopies from these pages.

Photocopying copyright material without permission is no different from stealing a magazine from a newsagent, only it doesn't seem like theft.
$T_{\text {he Copyright Licensing }}$ Agency (CLA) is an organisation which issues licences to bring photocopying within the law. It has designed licensing services

to cover all kinds of special needs in business, education, and government.

If you take photocopies from books, magazines and periodicals at work your employer should be licensed with CLA.

Make sure you are protected by a photocopying licence.

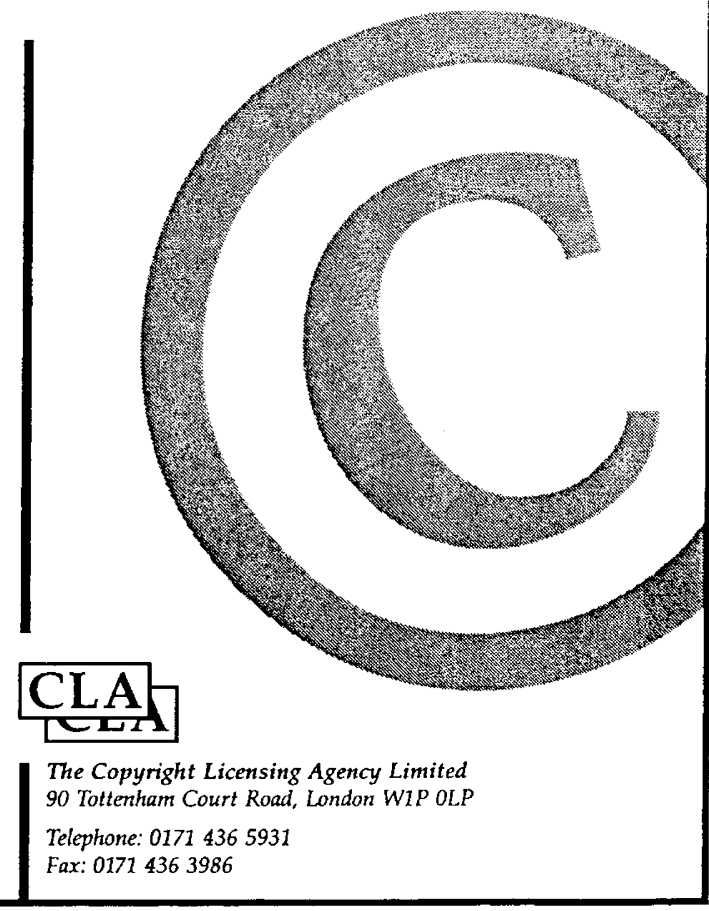

